\title{
The Psychological Aspect of Polypharmacy in Patients with Chronic Diseases. A Case Control Study about The Impact of Polypharmacy Regime Amongst Patients in Kurdistan Region of Iraq.
}

\section{Banan Rasool ( $\boldsymbol{\sim}$ bananqasm@gmail.com )}

Kurdistan Regional Government | Ministry of Health

\section{Shwan Amen}

Surgical Specialty Hospital, Cardiac Center, Erbil-Iraq

\section{Redar Amin}

Hawler Psychiatric Hospital, Erbil-Kurdistan Region of Iraq

\section{Gazang Abdullah}

Kurdistan Regional Government | Ministry of Health

\section{Ondřej Ferra}

Charles University

\section{Salah Hassan}

University of Kurdistan Hewler

\section{Elaf Othman}

Hawler Medical University

\section{Harez Fattah}

Hawler Medical University

\section{Payam Maaroof}

University of Jordan

Don Eliseo III Lucero-Prisno

London School of Hygiene \& Tropical Medicine

\section{Research Article}

Keywords: Polypharmacy, Psychological Impact, Chronic Disease, Mental Health, Medical Trauma

Posted Date: January 4th, 2022

DOI: https://doi.org/10.21203/rs.3.rs-1133431/v1

License: (c) (1) This work is licensed under a Creative Commons Attribution 4.0 International License. Read Full License 
Page $2 / 19$ 


\section{Abstract}

\section{Background:}

Patients on Polypharmacy regime due to having a chronic illness are at a higher risk of developing some form of negative psychological outcome as a result of their disease. This study aims to determine the impact of polypharmacy on the psychological aspects of a patient living with a chronic disease.

\section{Method:}

A case-control study conducted amongst patients in an outpatient clinic of an Internist (July 1st to September $1^{\text {st }}$, of 2021) in the city of Erbil, Iraq. Data was extracted from a self-assessed questioner. 200 participants were divided into polypharmacy (case group) and non-polypharmacy group (control group). Polypharmacy, being defined as the use of 5 or more medication which meant that participants in the case group must fit into these criteria. A 'Hospital Anxiety and Depression Scale' (HADS) was used to determine the presence of symptoms of depression and/or anxiety.

\section{Results:}

A totally of 200 patients were included in the study. Of these 100 participants were from the nonpolypharmacy group while the remaining 100 were from the polypharmacy group. Of these, depression among non-polypharmacy group (control) participants were $11 \%$ while $76 \%$ were found to be non-cases of depression. Compared to that, $31 \%$ of the polypharmacy group had depression while $51 \%$ were noncases for depression.

Also. the prevalence of depression and anxiety was increasing with an increase in number of medications taken by the patients. Depression was present in $11 \%$ of patients who took 4 or less medications while the prevalence increased to $21.1 \%, 34.9 \%$ and $42.1 \%$ in patients who took five, six to eight, and more than nine medications, respectively.

\section{Conclusion:}

There is a significant link between polypharmacy and psychological distress in patients with chronic diseases with a focus on depression and anxiety. Healthcare providers can help in detecting polypharmacy and in providing recommendations for simplifying medication regimes and reducing the outcomes of chronic diseases.

Trial registration: - This article doesn't contain any health care intervention on human participants.

\section{Background}

With the continuous rapid rise in both the prevalence and incidence of chronic diseases, the implementation of 'polypharmacy' has been used as a tool to withstand such dramatic growth ${ }^{[1]}$ 
In developed countries chronic conditions account for most of the primary care consultations, thus adding a bigger challenge to the health care system. ${ }^{[2]}$

Chronic diseases are the leading causes of death in the world. In 2016, it was responsible for $71 \%$ (41 million out of 57 million) of deaths worldwide ${ }^{[3]}$. The global prevalence of all the leading chronic diseases is increasing, with the majority occurring in developing countries ${ }^{[4]}$.

Polypharmacy according to the World Health Organization (WHO) is recognized as the concurrent use of multiple medications, routinely the use of five or more medications in order to manage a condition or multiple conditions. This includes over the counter medications, prescribed and/or traditional as well as complementary medication used by the patient ${ }^{[5]}$. Furthermore, the British Medical Journal (BMJ) defines polypharmacy as a cumulative use of five or more medications during a one-year period $[6,7,8]$.

Medical trauma: is clarified as a trauma that occurs due to any medical environment, the diagnostic and/or procedural experience that can have a powerful psychological impact due to patient's unique interpretation of the event ${ }^{[9]}$. It can also be experienced as a result of polypharmacy having lasting effects. While not a common term in the lexicon of health professionals, it is a phenomenon that deserves shedding light upon. Those who experience medical trauma can develop clinically significant psychological impact reactions such as anxiety, depression and somatic complaints. ${ }^{[9]}$

With that being said, numerous chronic diseases such as cardiovascular, diabetes, rheumatoid, thyroid diseases, kidney diseases, and many more conditions may require numerous medications to be prescribed as a means to improve the quality of life of the patient.

Appropriate polypharmacy usage recognizes that patients may benefit from multiple medications if the patient's clinical condition, comorbidities, allergy profile, potential drug-drug and drug-disease interactions are put into consideration ${ }^{[10]}$. However, bearing in mind that psychological aspects associated with polypharmacy as well as the adverse effects of these medications could potentially play a significant role in the determination of efficacy of the medications being prescribed. These adverse effects could also further increase the need to prescribe more medications in order to counteract them.

Multiple studies have suggested that there is a significant association linking polypharmacy with increase in incidence of psychological diseases.

In addition to increase in the risk of chronic disease diagnosis with age as well as medication usage for controlling their signs and improving patient's lifestyle ${ }^{[11,12,13]}$, studies have also shown that there is a chronological increase in mental diseases and the symptoms leading to them with aging. ${ }^{[14]}$

Despite this increase in prevalence of chronic diseases and multimorbiditices, little information exists on correlation of polypharmacy (using $5+$ medications). There is more information available regarding the link between polypharmacy and physical aspects of health than subjective ones. 
In this study we aim to further expand on the knowledge of this topic, including the incidence of different socioeconomic groups, genders, age groups and residential settings. ${ }^{[15,16]}$

In order to better understand the association between polypharmacy and its effects on mental health, it is important to take into consideration that there are wide ranges of presentations that could potentially fall under the umbrella of 'mental health'. Depression and neuropsychiatric symptoms such as anxiety and cognitive impairment are just a few of the symptoms that may appear amongst patients on polypharmacy regime. With a focus on rates of depression and anxiety in particular, this study captures a retrospective view and shines a light on the association of psychological aspects amongst polypharmacy patients with chronic conditions.

\section{Methods}

\section{Design and settings:}

A case-control study conducted amongst patients in an outpatient clinic of an Internist from July 1st to September 1 st of 2021 in the city of Kurdistan, Iraq.

\section{Sample Size and Selection:}

Data were collected from 200 patients, divided in to two groups each consisting of 100 patients.

The first group was the non-polypharmacy group (control group), including patients with chronic diseases, using less than five different medications.

The second group consisted of patients with chronic diseases using five or more medications, known as the polypharmacy group (case group).

The number of medications were classified as either non-polypharmacy (which was mainly for the control group, who regularly took 4 or less medications) and polypharmacy (for the case group who took 5 or more medications on a regular base). The polypharmacy group were further categorized into different variables, such as 5 medications, 6-8 medications, and use of 9 or more medications.

Exclusion criteria for both groups were the presence of renal failure patients on dialysis, known case of heart failure, or any end stage organ disease, any known case of psychiatric illness or on antidepressants/anti-psychotic medications usage.

\section{Data Collection:}

Data were obtained through completion of questionnaires, filled out by the well trained personals through a face to face interview for each participant. Participants were selected specifically according to the exclusion criteria and after clarifying the purpose of the study and receiving both verbal and written 
consent from the participants, they were notified that they were able to withdraw from the study at any stage or time.

A specifically designed questionnaire was used, consisting of three parts.

- The first part focusing on patient's identity, such as age, gender, presence of risk factors for chronic disease such as hypertension, ischemic heart disease, diabetes and others.

- The second part, a Hospital Anxiety and Depression Scale (HADS) was used to determine the presence of symptoms of depression and/or anxiety. The HADS is a questionnaire that has been found to be a reliable instrument for detecting states of anxiety and depression in the setting of hospital outpatient clinics.

- And lastly, patients were graded based on the scores they have reached in the HADS. Patients scoring less than 8 points were ruled as non-cases, patients scoring 8 to 10 points as borderline cases and patients scoring more than 10 points as cases. The grading and scoring were conducted separately for depression and anxiety. ${ }^{(17,18)}$

\section{Statistical analysis of data:}

The statistical analysis was done using IBM SPSS Statistics Ver. 28.0, IBM Corp., Armonk NY. Baseline patient characteristics were summarized. All data were presented as $n(\%)$ for categorical variables. Comparisons between groups were made using Chi-square tests. A P $<0.05$ was considered statistically significant.

\section{Ethical consideration}

The study was conducted following the ethical principles origin in the declaration of Helsinki. The study protocol and the subject's information and consent forms were reviewed and confirmed by the local ethics committee of Hawler Medical University.

\section{Results}

The study has enrolled 200 participants who were interviewed. The sample size was divided into two groups, 100 were non polypharmacy group and the other 100 were patients who were among the polypharmacy group.

The general median age for the sample size was 59 years $(S D=+-12.7)$, the maximum age was 88 years, the minimum 20 years. $28.5 \%$ (57) were between the ages of 50 to $59,27.5 \%$ (55) were between 60 to 69 , $22.5 \%$ (45) were 70 years and older. This is summarized in Figure 1. 50.5\% (101) of the participants were male and the remaining $49.9 \%$ (99) were females.

Among the participants, the prevalence of hypertension was 71.5\% (143), and 61.5\% (123) were diagnosed with ischemic heart disease, $38.5 \%$ (77) and 14.5\% (29) were known cases of diabetes 
mellitus and had some form of arrhythmia respectively. While a smaller number of participants had rheumatoid 7.5\% (15) and Thyroid 3.75\% (7.5) diseases. Keeping in mind that many of the patients interviewed presented with multiple numbers of chronic diseases rather than a single chronic condition. As summarized in Figure 2.

In the non-polypharmacy group, all participants were taking four or less medications, however among the polypharmacy group, 38\% (38) of participants were receiving five medications, 43\% (43) were receiving 6 to 8 medications and 19\% (19) nine or more medications. This is summarized in Figure 3.Regarding the duration of medication's usage, $45 \%$ (90) of the participants were taking these medications on an average period of two to nine years.

Based on the data obtained it was quite evident that both the non-polypharmacy group and the polypharmacy group presented with some form of similar family history of grief. The prevalence in the non-polypharmacy group was $37 \%$, while in the polypharmacy group was $38 \%$.

The prevalence of depression among non-polypharmacy group (control) participants were $11 \%(11), 13 \%$ (13) cases were borderline. The remaining $76 \%$ (76) were found to be non-cases of depression, according to the used HADS scoring system. Compared to that, $31 \%$ (31) of the polypharmacy group had depression, $18 \%$ (18) were borderline and the remaining $51 \%$ (51) were non-cases for depression. This finding was statistically significant with $P<0.001$. Summarized in Figure 4.

As for anxiety, in the non-polypharmacy group, 7\% (7) were found to be cases, $16 \%$ (16) were borderline and $77 \%$ (77) were non-cases for anxiety. In the polypharmacy group, $19 \%$ (19) had anxiety, $15 \%$ (15) were borderline and the remaining $66 \%$ (66) were found to be non-cases for anxiety. This finding was statistically significant with $P=0.046$. This is summarized in Figure 5 .

One of the interesting points to be taken into consideration was, the prevalence of depression and anxiety was increasing with an increase in number of medications taken by the patients, in other words, the higher the number of medications used, the higher the prevalence of psychological symptoms were noticed among the participants.

Depression was present in $11 \%$ of patients who took 4 or less medications (the non-polypharmacy group). The prevalence increased to $21.1 \%, 34.9 \%$ and $42.1 \%$ in patients who took five, six to eight, and more than nine medications, respectively. This finding was statistically significant with $P=0.003$. This is summarized in Figure 6.

Anxiety was present in $7 \%$ of patients who took 4 or less medications (the non-polypharmacy group). The prevalence increased to $15.8 \%, 16.3 \%$ and $31.6 \%$ in patients who took five, six to eight, and more than nine medications, respectively. However, this finding was not statistically significant with $P=0.08$.

Regarding the frequency of Depression for both genders, it was noticed that there was a higher proportion of participants with depression among the polypharmacy group than the non-polypharmacy group. 
specifically, $7.4 \%$ of male participants in the non-polypharmacy group had depression. This frequency increased to $23.4 \%$ in the polypharmacy group. This finding was statistically significant with $P=0.024$. regarding the female participants, $15.2 \%$ of them in the non-polypharmacy group had depression and frequency has increased to $37.7 \%$ among the polypharmacy group. This finding was statistically significant with $P=0.028$. This is summarized in Figure 7.

Anxiety, which was one of the psychological presentations that was focused in our study, had higher frequencies among the polypharmacy group in both genders.

Among male patients, $1.9 \%$ in the non-polypharmacy group had anxiety. And this has increased to $8.5 \%$ among the polypharmacy group. However statistically this finding was not significant with $P=0.356$.

Among female participants, $13 \%$ of the non-polypharmacy group had anxiety comparing to the $28.3 \%$ of the polypharmacy group. This finding was not statistically significant with $P=0.199$.

Hypertension as a specific entity, was closely related to anxiety among participants, $17.5 \%$ of hypertensive patients were noted to have anxiety according to the HADS scoring, and this finding was statistically significant with $\mathrm{P}=0.008$.

Patients who were suffering from different forms of cardiological arrhythmias also noted to be more anxious than others, with $17.2 \%$ of the cases having anxiety according to the scoring system of HADS, and this finding was statistically significant with $P=0.021$.

Diabetic patients tended to present with more psychological symptoms, the frequency of depression and anxiety among this specific group of participants were found to be $35.1 \%$ and $14.3 \%$ respectively. These findings were statistically significant with $P<0.001$ and $P=0.038$ respectively.

Focusing on patients with rheumatoid diseases, a great proportion of them $(40 \%)$ had depression. And this finding was statistically significant with $P=0.01$.

This is summarized in Table 1 and Table 2 for both anxiety and depression frequency among patients with specific chronic diseases. 
Table 1

Frequency of Anxiety among Patients with Different Risk

Factors and Their P Values.

\begin{tabular}{|lll|}
\hline Risk factor & $\begin{array}{l}\text { Prevalence of } \\
\text { anxiety }\end{array}$ & $P$ value \\
\hline Hypertension & $17.5 \%$ & $P=0.008$ \\
\hline $\begin{array}{l}\text { Arrhythmia } \\
\text { Diseases }\end{array}$ & $17.2 \%$ & $P=0.021$ \\
\hline $\begin{array}{l}\text { Diabetes Mellitus } \\
\begin{array}{l}\text { Rheumatoid } \\
\text { Disease }\end{array}\end{array}$ & $14.3 \%$ & $P=0.038$ \\
\hline
\end{tabular}

Table 2

Frequency of Depression among Patients with Different Risk Factors and Their P Values.

\begin{tabular}{|lll|}
\hline Risk factor & Prevalence of depression & P value \\
\hline Hypertension & $24.5 \%$ & $P=0.078$ \\
\hline Arrhythmia Diseases & $31 \%$ & $P=0.217$ \\
\hline Diabetes Mellitus & $35.1 \%$ & $P<0.001$ \\
\hline Rheumatoid Disease & $40 \%$ & $P=0.01$ \\
\hline
\end{tabular}

\section{Discussion}

There have been various studies focusing on the association between polypharmacy and their physical aspects of health with some concentrating on the psychological aspects of patients with chronic diseases. However, according to the available data, this is one of the first studies that was done in the region that demonstrates this association amongst medically treated patients that also presented with depression and anxiety symptoms. From the 200 patients chosen randomly from the outpatient clinic of an Internist, polypharmacy has been counted as one of the contributing factors of negative mental health outcome amongst these patients.

In other studies polypharmacy has been identified as one of the negative health outcomes including dementia ${ }^{[19]}$ and death. ${ }^{[20]}$ The mechanism for the association is not clearly elucidated but can involve an increase in drug-related problems. ${ }^{[20]}$

During this study it was significantly clear that the prevalence of depression and anxiety increased in patients with polypharmacy with chronic diseases. Similar multiple other studies have identified a positive association between the number of medications used by patients with chronic medical diseases 
and psychological symptoms. Furthermore, a study in 2017 revealed that depressive disorders accounted for approximately $3 \%$ of all disability-adjusted life-years worldwide. ${ }^{\text {[21] }}$

Regardless of gender, prevalence of depression increases by $20 \%$ when polypharmacy had been implemented in this study. The number of borderline cases also increases by $5 \%$. These results are consistent with a systematic review and meta-analysis conducted by Sirinoot and Janthima in 2021 [15]. Accordingly, when polypharmacy was defined as the number of medications used concurrently, an increase in the number of drugs was associated with an increased risk of depression by $55 \%$. The study extended their findings when polypharmacy was defined as the use of 5 or more medication, the risk of depression increased to $73 \%$. Further clarifying that polypharmacy had a strong association with the number of medications that patients have been using.

It was also demonstrated during this study that the prevalence of depression in females is significantly higher than in males. This difference was even more pronounced when polypharmacy regime has been implemented. Our study showed that amongst male patients, $7.4 \%$ (4) in the non-polypharmacy group had depression and this number increased to $23.4 \%(11)$ in the cases group. Meanwhile in females, $15.2 \%$ (7) of patients in the control group had depression. With an increase to $37.7 \%(20)$ in the cases group. Our data were also consistent with research conducted amongst US-born Mexican American older adults in 2019. It showed that the prevalence of depression was higher amongst female patients on polypharmacy compared to that in males. ${ }^{[16]}$ Some sources suggest that females in general are more prone to psychiatric disorders than men. This could be due to regular hormonal fluctuations, as well as other biological factors increasing their risk for depression. ${ }^{22]}$

We documented a link between polypharmacy and distress above all confounders, including age. Higher age groups have shown to be a risk factor of polypharmacy, simply because aging is a process associated with the development of multiple chronic medical conditions (multimorbidity) that need pharmacological treatment ${ }^{[23]}$. Most people in their sixties have multimorbidity ${ }^{[24]}$. As these conditions are diagnosed, a person's risk of polypharmacy increases ${ }^{[25]}$.

When it comes to categorizing the chronic diseases in this study it is noticed that in the case group (those on polypharmacy regime) the minimum number of medications taken by patients with chronic diseases is $5(38 \%)$ while $43(43 \%)$ of patients are taking 6-8 medications showing that the number of the medication's usage increases with the rise in patients complaining of number of chronic diseases and thus leading to the demand for multiple medications to be administrated at one time. Similarly, from research conducted in 2012. It was found that the highest rate of polypharmacy (64.1\%) was found in sample individuals with all three (cardiometabolic, respiratory and musculoskeletal) disease clusters. The next highest rates $(41.2 \%$ and $41.8 \%$ ) were observed among those with cardiometabolic and musculoskeletal disease clusters and among those with cardiometabolic and respiratory disease clusters. The lowest rates were found in those with only musculoskeletal (7.9\%) and only respiratory clusters $(7.2 \%){ }^{[26]}$ Suggesting that with greater number of comorbidities the number of medications used will also dramatically increases. 
The results from this research also revealed that the presence of a particular risk factor can have an independent effect on presence of depression and anxiety. Of the patients complaining from diabetes in the case group $35.1 \%$ of them had depression and $14.3 \%$ had anxiety. This could be explained by the lifestyle modifications needed for the treatment, constant worry of complications or the influence of the disease and its treatments on patient's social life. These findings are consistent with previous research conducted in Saudi Arabia, which found that $37 \%$ of diabetics to have depression. ${ }^{[27,28]}$

A similar effect was observed in patients with rheumatoid disease (RD). In this study, $40 \%$ of patients with rheumatoid disease were found to have depression. More recent studies have shown that systemic inflammation conditions in particular RD, measured by acute-phase reactants and proinflammatory cytokines, are often associated with the development of depression [29-31], and it has been suggested that systemic inflammation may be associated with, cause, or contribute to depressive symptoms during disorders of chronic inflammation ${ }^{[32-34]}$ thus making the incidence of depression substantially higher amongst patients diagnosed with RD.

A risk factor linked to increase in incidence of anxiety is hypertension, which was noticed to be in about $24.5 \%$ of our participants. Hypertension is a condition that requires a dramatic lifestyle modification as well as proper compliance to medication in order to control the condition. Adherence to medication may be a contributing factor to anxiety and patients may constantly be worrying about the proper administration or constant adjusting of lifestyle in order to control their blood pressure. One study noted that anxiety was common among $225(57 \%)$ of the hypertensive patients. This result corroborates the high prevalence of anxiety found among hypertensive patients in varied countries such as South Africa, China and Argentina [35,36,37]; thus showing the presence of anxiety in hypertension in different countries depending on the level of patient education and early detection methods implemented by such countries.

\section{Limitations}

Some limitations of our study need to be taken into account while interpreting the findings. All measures in the study were self-reported and subject to recall bias. This study is an observational study and may suffer from selection biases with a sample of 200 participants. Additionally, we included only individuals who were attending the private clinic of an Internal medicine physician who was specialized in the field of Cardiology, thus making our sample less heterogeneous. Most of the participants had some form of history of cardiovascular disease. We did not measure severity of chronic conditions which may necessitate multiple medications use, also we did not categories the medications usage based on time, rather we broadly mentioned that they were between 2-9 years. Polypharmacy was also abundantly defined as number of therapeutic classes and in many of the chronic condition's combination therapies have become the norm rather than an exception.

Future studies could supplement patients' report on adherence with objective assessments of adherence.

\section{Conclusion}


To summarize, this study proposed a link between polypharmacy and psychological distress in patients with chronic diseases with a focus on depression and anxiety. Thus, although Polypharmacy may be needed by patients to manage chronic diseases, it also signifiantly affects the mental health of the patients. There is a need for effective interventions that jointly evaluate inappropriate medication use, physical health, as well as the psychological distress amongst patients with chronic diseases.

\section{Abbreviations}

HADS: Hospital Anxiety and Depression Scale

RD: Rheumatoid Disease

WHO: World Health Organization

BMJ: British Medical Journal

\section{Declarations}

Ethics Approval and Consent to participate the study was conducted in accordance with the ethical principles that have their origin in the Declaration of Helsinki. It was carried out with the patient's verbal/written consent. Informed consent has been taken from all participants. The study protocol, the methodology, the subject information, and the consent form were reviewed and approved by the Ethics Committee of Hawler Medical University (HMU-MC6-PC8)

Consent for publication: not applicable

Availability of data and materials: All data generated or analyzed during this study are included in this published article and its supplementary information files.

Competing interests: The authors declare that they have no competing interests,

Funding: not funded.

Authors' contributions:

BR., SA., RA. and SH. contributed to the conception and design of the study. OF., GA., PM and BR. were in charge of the data analysis and interpretation as well as the writing of the paper. HF and EO. Contributed to the acquisition of the data. BR., G.A. and DP. revised the manuscript. All authors read and approved the final manuscript.

\section{Acknowledgements}

We would like to thank all the staff of Dr Shwan Amen's outpatient clinic, who were very cooperative and helpful, and made the data collection and patient selection process much easier. As well as all the 
participants who allocated their time to the contribution to the discovery of wonderful and interesting facts, we were able to obtain.

\section{References}

1. -Nobili A, Garattini S, Mannucci PM. Multiple diseases and polypharmacy in the elderly: challenges for the internist of the third millennium. J Comorb. 2011;1:28-44. Published 2011 Dec 27. doi:10.15256/joc.2011.1.4 rwjf54583 (1).pdf

2. -Goodwin N, Lawton-Smith S. Integrating care for people with mental illness: the Care Programme Approach in England and its implications for long-term conditions management. Int $\mathrm{J}$ Integr Care. 2010;10:e040. Published 2010 Mar 31. doi:10.5334/ijic.516

3. -Global Health Estimates 2016: Deaths by Cause, Age, Sex, by Country and by Region, 2000-2016. Geneva: World Health Organization; 2018.

4. -Yach, D., Hawkes, C., Gould, C. L., \& Hofman, K. J. (2004). The global burden of chronic diseases: overcoming impediments to prevention and control. JAMA, 291(21), 2616-2622. https://doi.org/10.1001/jama.291.21.2616

5. -Masnoon, N., Shakib, S., Kalisch-Ellett, L., \& Caughey, G. E. (2017). What is polypharmacy? A systematic review of definitions. BMC geriatrics, 17(1), 230. https://doi.org/10.1186/s12877-0170621-2

6. -Slabaugh, S. L., Maio, V., Templin, M., \& Abouzaid, S. (2010). Prevalence and risk of polypharmacy among the elderly in an outpatient setting: a retrospective cohort study in the Emilia-Romagna region, Italy. Drugs \& aging, 27(12), 1019-1028. https://doi.org/10.2165/11584990-00000000000000

7. -Monégat $M$, Sermet $C$, Perronnin $M$, et al. Polypharmacy: Definitions, measurement and stakes involved. Review of the literature and measurement tests. Quest d'économie la santé 2014;204:1-8.

8. -Grimmsmann T, Himmel W. Polypharmacy in primary care practices: an analysis using a large health insurance database. Pharmacoepidemiol Drug Saf. 2009;18(12):1206-1213. doi:10.1002/pds.1841

9. -Michelle Flaum Hall, E. L.-S., \& Scott E. Hall, P. L.-S. (2017). Managing the Psychological Impact of Medical Trauma: A Guide for Mental Health and Health Care Professionals. Springer Publishing Company.

10. -Cadogan CA, Ryan C, Hughes CM. Appropriate Polypharmacy and Medicine Safety: When Many is not Too Many. Drug Saf. 2016;39(2):109-116. doi:10.1007/s40264-015-0378-5

11. -Flynn MG, Markofski MM, Carrillo AE. Elevated Inflammatory Status and Increased Risk of Chronic Disease in Chronological Aging: Inflamm-aging or Inflamm-inactivity?. Aging Dis. 2019;10(1):147156. Published 2019 Feb 1. doi:10.14336/AD.2018.0326

12. -Nyberg ST, Singh-Manoux A, Pentti J, et al. Association of Healthy Lifestyle With Years Lived Without Major Chronic Diseases. JAMA Intern Med. 2020;180(5):760-768. 
doi:10.1001/jamainternmed.2020.0618

13. -Gao L, Maidment I, Matthews FE, Robinson L, Brayne C; Medical Research Council Cognitive Function and Ageing Study. Medication usage change in older people (65+) in England over 20 years: findings from CFAS I and CFAS II. Age Ageing. 2018;47(2):220-225. doi:10.1093/ageing/afx158

14. -Skoog I. Psychiatric disorders in the elderly. Can J Psychiatry. 2011;56(7):387-397. doi:10.1177/070674371105600702

15. -Palapinyo, S., Methaneethorn, J., \& Leelakanok, N. (2021). Association between polypharmacy and depression: a systematic review and meta-analysis. Journal of Pharmacy Practice \& Research, 1. https://doi.org/10.1002/jppr.1749

16. -Assari, S., Wisseh, C., Saqib, M., Helmi, H., \& Bazargan, M. (2019). Polypharmacy and Depressive Symptoms in U.S.-Born Mexican American Older Adults. Psych, 1(1), 491-503. https://doi.org/10.3390/psych1010038

17. -Snaith R. P., \& Zigmond A. S. (1986). The Hospital Anxiety And Depression Scale. British Medical Journal (Clinical Research Edition), 292(6516), 344.

18. -Bjelland, I., Dahl, A. A., Haug, T. T., \& Neckelmann, D. (2002). The validity of the Hospital Anxiety and Depression Scale: An updated literature review. Journal of Psychosomatic Research, 52(2), 69-77. https://doi.org/10.1016/S0022-3999(01)00296-3

19. -Liu Q, He H, Yang J, Feng X, Zhao F, Lyu J. Changes in the global burden of depression from 1990 to 2017: Findings from the Global Burden of Disease study. J Psychiatric Res. 2020; 126: 134-40.

20. -Leelakanok N, Holcombe AL, Lund BC, Gu X, Schweizer ML. Association between polypharmacy and death: A systematic review and meta-analysis. J Am Pharm Assoc. 2017; 57(6): 729-38.e10.

21. -Leelakanok N, D'Cunha RR. Association between polypharmacy and dementia - A systematic review and metaanalysis. Aging Ment Health. 2019; 23(8): 932-41.

22. -Platt JM, Bates LM, Jager J, McLaughlin KA, Keyes KM. Changes in the depression gender gap from 1992 to 2014: Cohort effects and mediation by gendered social position. Soc Sci Med. 2020;258:113088. doi:10.1016/j.socscimed.2020.113088

23. -Pierce, M.B.; Silverwood, R.J.; Nitsch, D.; Adams, J.E.; Stephen, A.M.; Nip, W.; Macfarlane, P.; Wong, A.; Richards, M.; Hardy, R.; et al. Clinical disorders in a post war British cohort reaching retirement: Evidence from the first National Birth Cohort study. PLoS ONE 2012, 7, e44857.

24. -Kuh, D.; Wong, A.; Shah, I.; Moore, A.; Popham, M.; Curran, P.; Davis, D.; Sharma, N.; Richards, M.; Stafford, M.; et al. The MRC National Survey of health and development reaches age 70: Maintaining participation at older ages in a birth cohort study. Eur. J. Epidemiol. 2016, 31, 1135-1147.

25. -McIntyre, R.S.; Jerrell, J.M. Polypharmacy in children and adolescents treated for major depressive disorder: A claims database study. J. Clin. Psychiatry 2009, 70, 240-246

26. -Vyas A, Pan X, Sambamoorthi U. Chronic Condition Clusters and Polypharmacy among Adults. Int J Family Med. 2012;2012:193168. doi:10.1155/2012/193168 
27. -J. M. Madkhali, A. A. Hakami, A. H. Dallak et al., "Prevalence and associated factors of depression among patients with diabetes at Jazan Province, Saudi Arabia: a cross-sectional study," Psychiatry Journal, vol. 2019, 6 pages, 2019.

28. -Norah Muqbil Alhunayni, Amal Elwan Mohamed, \& Sabry Mohamed Hammad. (2020). Prevalence of Depression among Type-II Diabetic Patients Attending the Diabetic Clinic at Arar National Guard Primary Health Care Center, Saudi Arabia. Psychiatry Journal, 2020. https://doi.org/10.1155/2020/9174818

29. -Capuron L, Dantzer R. Cytokines and depression: the need for a new paradigm. Brain Behav. Immun. 2003; 17 Suppl. 1:S119-S124. [PubMed: 12615197]

30. -Howren MB, Lamkin DM, Suls J. Associations of depression with C-reactive protein, IL-1, and IL-6: a meta-analysis. Psychosom. Med. 2009; 71(2):171-186. [PubMed: 19188531]

31. -Irwin MR, Miller AH. Depressive disorders and immunity: 20 years of progress and discovery. Brain Behav. Immun. 2007; 21(4):374-383. [PubMed: 17360153]

32. -Dantzer R, O'Connor JC, Freund GG, Johnson RW, Kelley KW. From inflammation to sickness and depression: when the immune system subjugates the brain. Nat. Rev. Neurosci. 2008; 9(1):46-56. [PubMed: 18073775]

33. -Miller AH, Maletic $\mathrm{V}$, Raison CL. Inflammation and its discontents: the role of cytokines in the pathophysiology of major depression. Biol. Psychiatry. 2009; 65(9):732-741. [PubMed: 19150053]

34. -Evans DL, Charney DS, Lewis L, et al. Mood disorders in the medically ill: scientific review and recommendations. Biol. Psychiatry. 2005; 58(3):175-189. [PubMed: 16084838]

35. -Vetere G, Ripaldi L, Ais E, Korob G, Kes M, Villamil A: Prevalence of anxiety disorders in patients with essential hypertension. Vertex 2007, 18(71):20-25.

36. -Grimsrud A, Stein D, Seedat S, Williams D, Myer L: The association between hypertension and depression and anxiety disorders: results from a nationally representative sample of South African adults. PLoS One 2009, 4:2.

37. -Han J, Yin XM, Xu F, Hong X, Liang YQ, Wang ZY: A case-control study on depression and anxiety in hypertensive patients. Chin J Epid 2009, 29(2):125-127

\section{Figures}




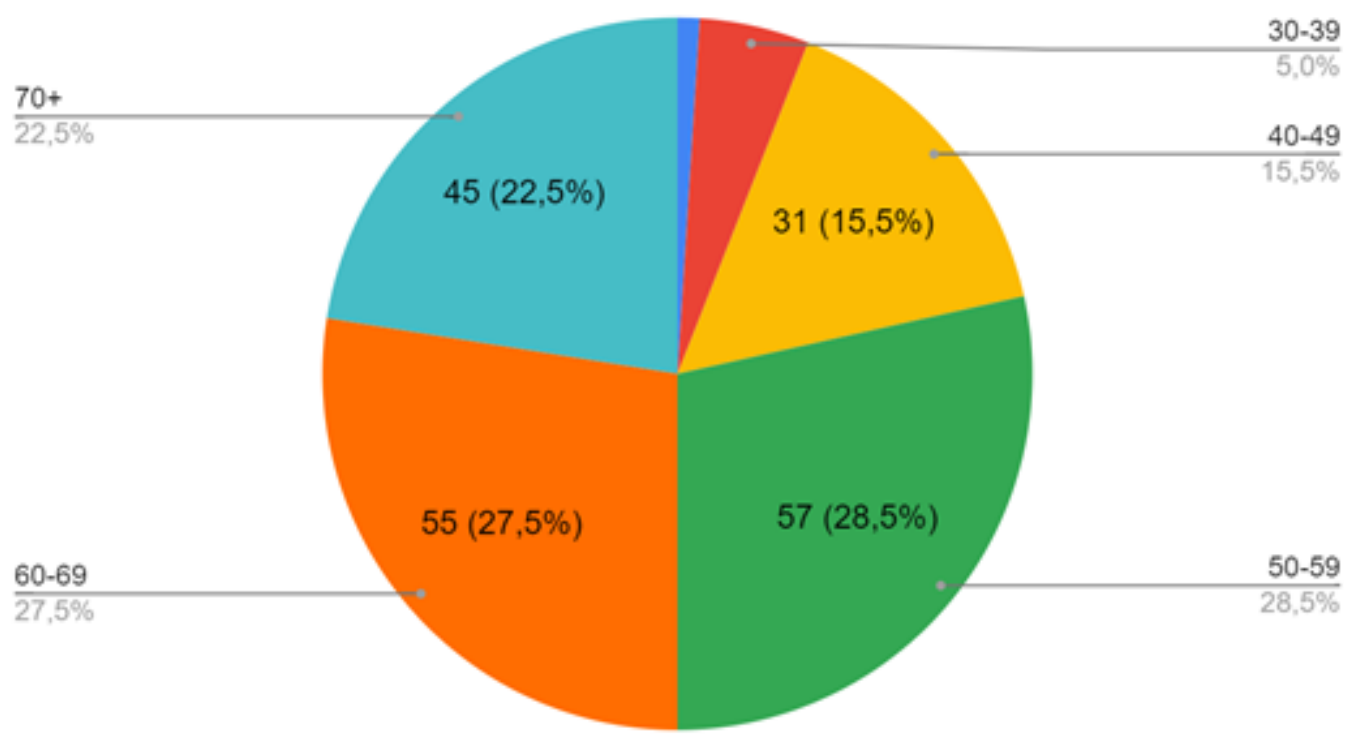

Figure 1

Shows the age distribution amongst participants.

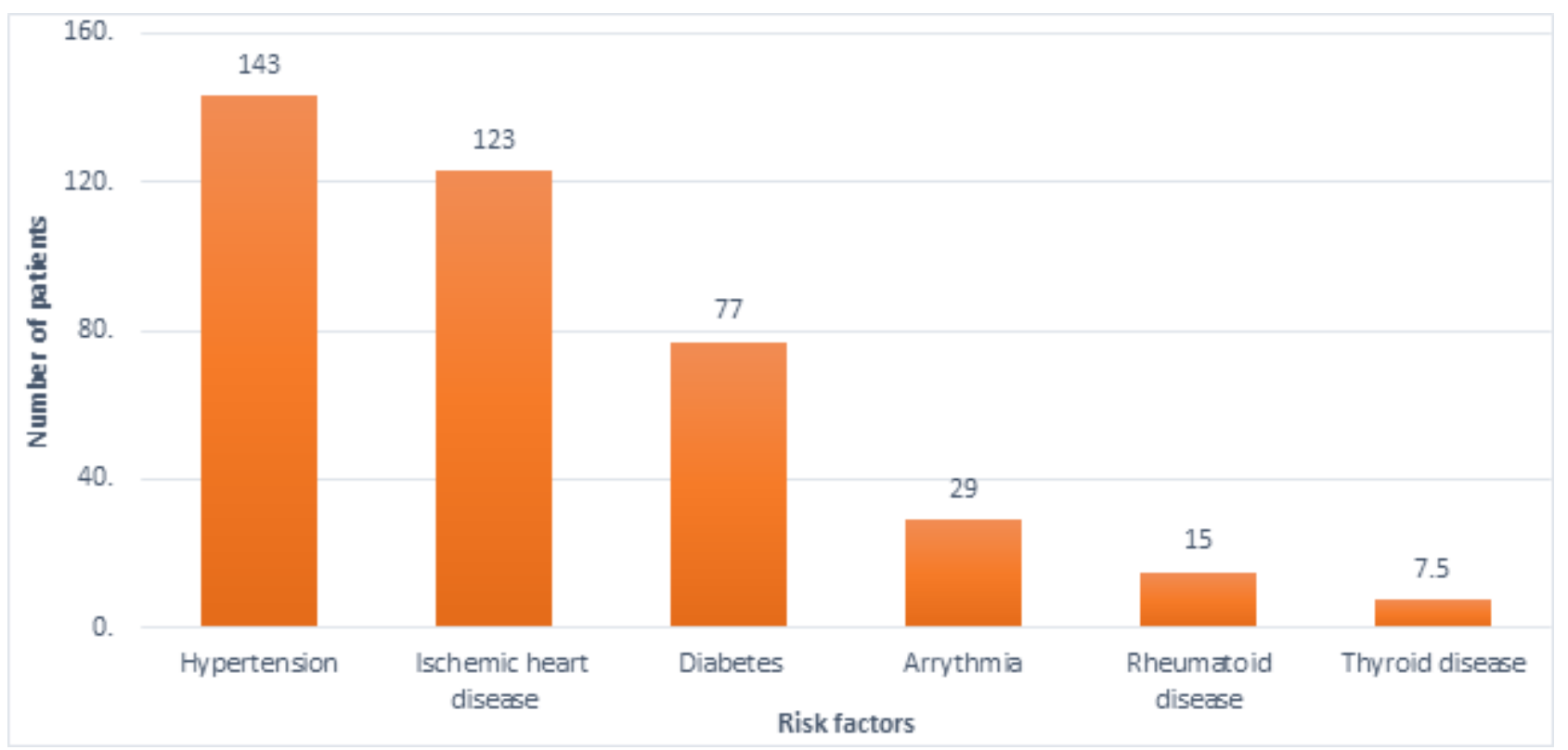

\section{Figure 2}

Shows the prevalence of risk factors among included participants 


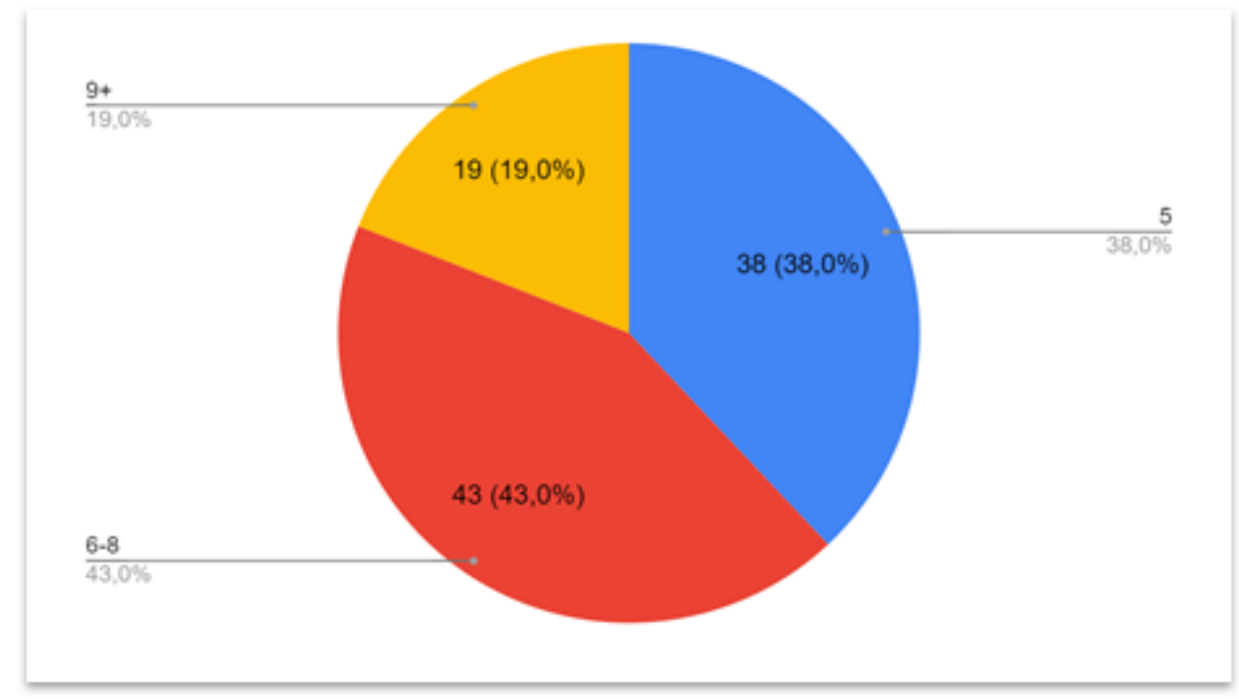

\section{Figure 3}

Shows the frequency of medication's usage taken by participants (Polypharmacy group).

63

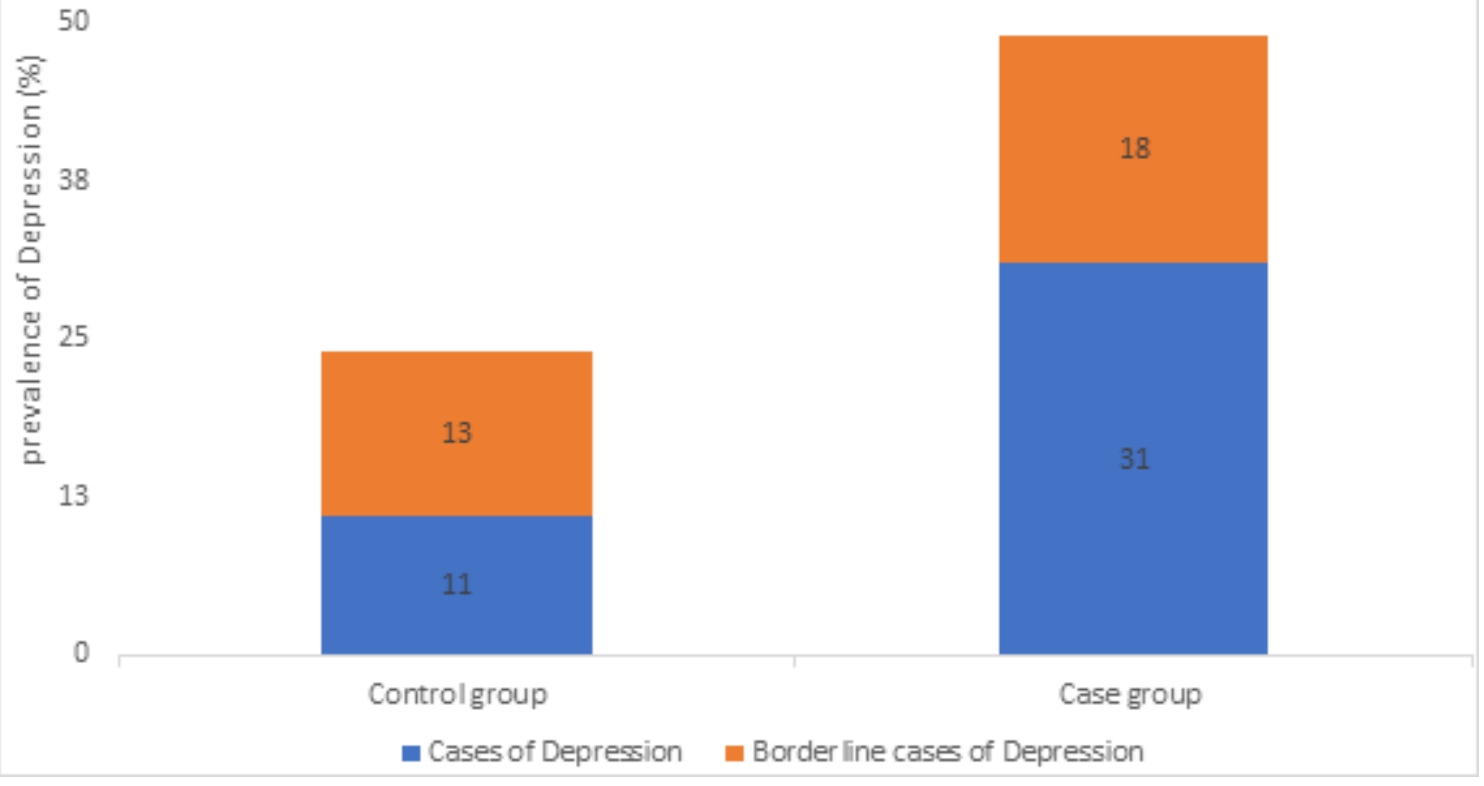

Figure 4

Frequency of depression among participants on polypharmacy regime and those who are not on polypharmacy regime 


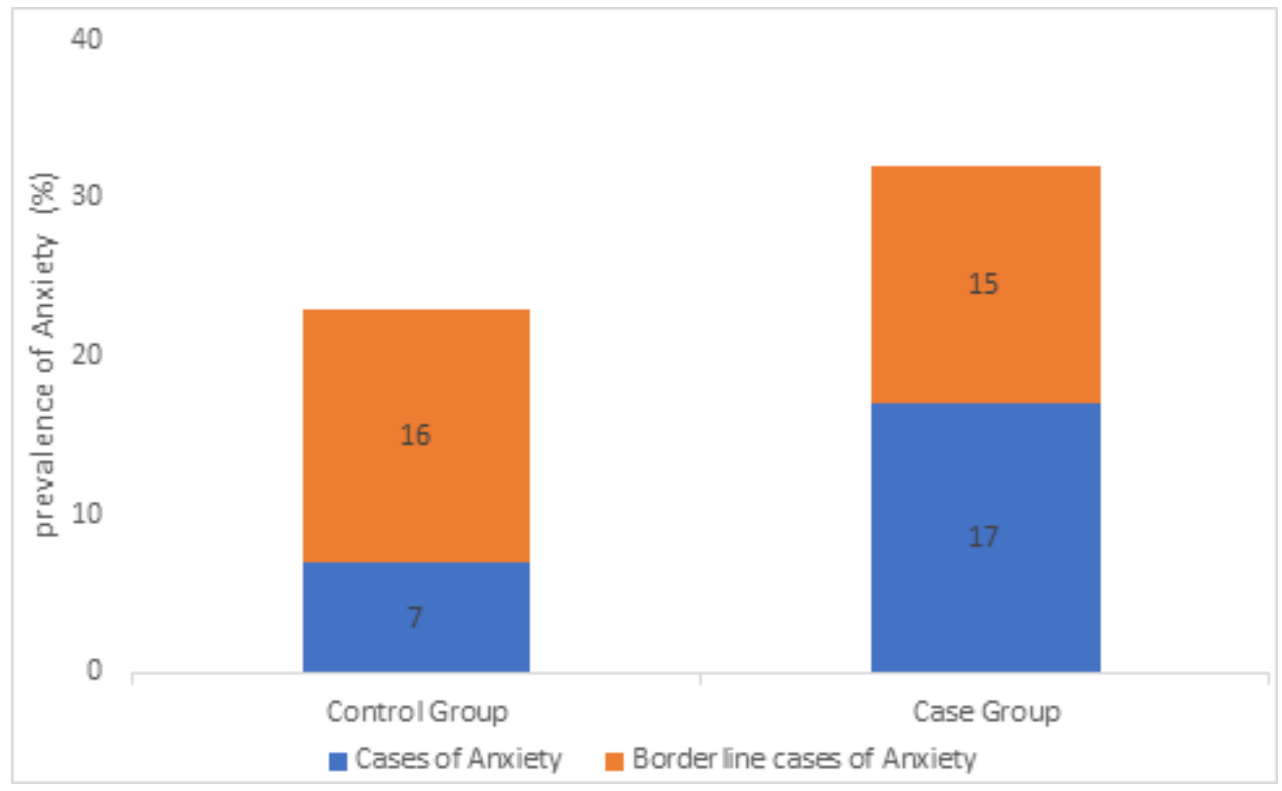

Figure 5

Frequency of anxiety amongst participants on polypharmacy regime and those who are not on polypharmacy regime.

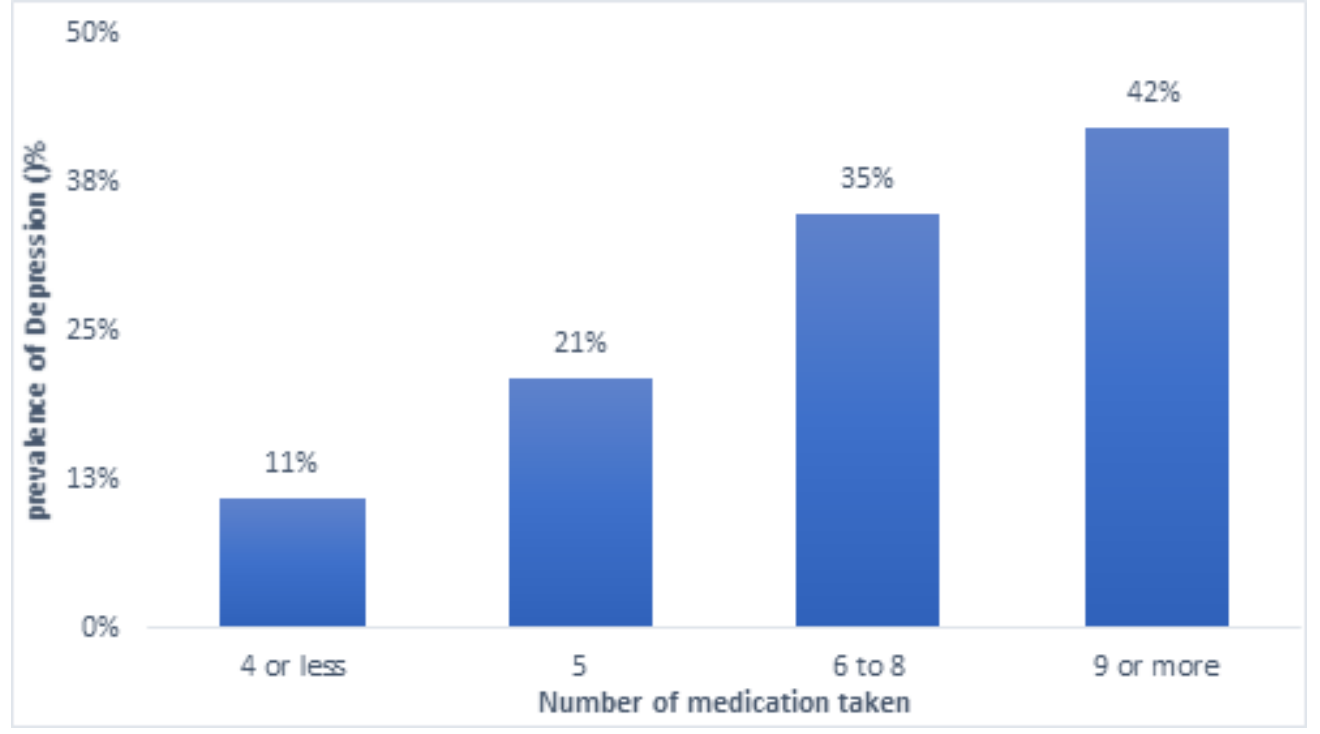

Figure 6

Prevalence of Depression amongst patients taking medications for multiple chronic diseases. 
$40 \%$

$30 \%$

岌 $20 \%$

$10 \%$

$0 \%$
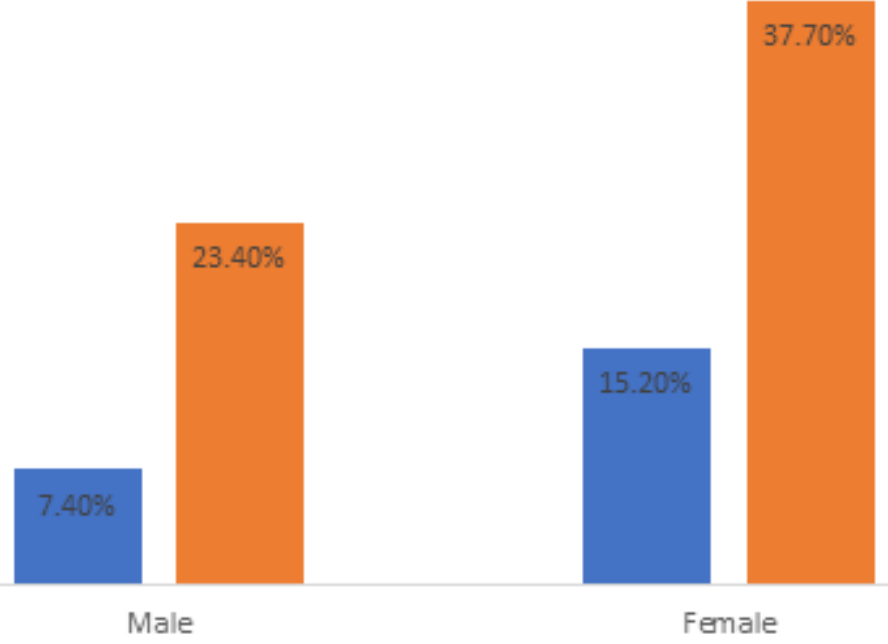

Female

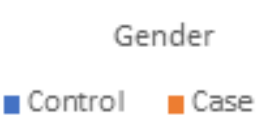

Figure 7

Shows the Frequency of depression among different genders. 